\title{
Correlation of cognitive and masticatory function in Alzheimer's disease
}

\author{
Camila Heitor Campos ${ }^{1}$ - Giselle Rodrigues Ribeiro ${ }^{1}$. José Luiz Riani Costa ${ }^{2}$. \\ Renata Cunha Matheus Rodrigues Garcia ${ }^{1}$
}

Received: 22 January 2016 / Accepted: 21 July 2016 /Published online: 30 July 2016

(C) Springer-Verlag Berlin Heidelberg 2016

\begin{abstract}
Objectives This study investigated chewing function in elderly individuals with Alzheimer's disease (AD) and correlated chewing function with cognitive status.

Materials and methods Sixteen elderly individuals with mild AD (mean age $76.7 \pm 6.3$ years; 8 men, 8 women) and 16 age and gender-matched healthy controls (mean age $75.23 \pm 4.4$ years; 8 men, 8 women) were included in this study. All volunteers wore removable prostheses: 11 were totally edentulous and five were partially edentulous in each group. Chewing function was evaluated via masticatory performance (MP) using Optocal chewable test material and a sieve fractionation method. Cognitive functioning was assessed by the Mini Mental State Exam (MMSE), administered by a trained examiner. Data were analyzed by non-paired $t$ test and Pearson's correlation with $\alpha=0.05$.

Results Compared to controls, mild $\mathrm{AD}$ patients had decreased MP $(P<0.01)$ and MMSE $(P=0.01)$. MP showed a moderate negative correlation with MMSE $(r=-0.69)$.

Conclusions Mild AD was associated with impaired chewing function.

Clinical relevance Knowledge that mild AD has an impact on chewing is important for dental professionals in decisionmaking related to prosthetics and general dental treatment.
\end{abstract}

Keywords Alzheimer's disease · Mastication · Cognition

Renata Cunha Matheus Rodrigues Garcia regarcia@ fop.unicamp.br

1 Department of Prosthodontics and Periodontology, Piracicaba Dental School, University of Campinas, Avenida Limeira, no. 901, Bairro Areião, Piracicaba, SP CEP: 13414-903, Brazil

2 Department of Physical Education, Paulista State University Julio de Mesquita Filho, Sao Paulo, Brazil

\section{Introduction}

Alzheimer's disease is the leading cause of dementia affecting the elderly population [1], causing progressive decline of cognitive functions with consequent impairment of memory, learning, and communication [2]. Loss of functionality has a great impact on affected individuals, leading to loss of autonomy and requiring assistance to perform activities of daily living [3].

Cognitive decline is due to brain neurophysiological changes, such as diffuse cortical atrophy and loss of neurons, cell groups, and synapses [3]. Moreover, intraneuronal alterations occur, including extracellular deposits of beta-amyloid protein and formation of neuritic plaques [4-6]. These changes take place in brain regions involved in learning, memory, and emotional behavior [7], areas that are also known to be related to chewing function $[8,9]$.

The mechanisms underlying dementia are not fully understood, but some risk factors have been identified [8, 10, 11], such as age, female gender, heritage, presence of ApoE e4 allele, education and occupation level, depression, head trauma, alcohol abuse, and atherosclerosis [10, 12]. In addition, epidemiological studies have indicated that having few teeth is a risk factor for the development of dementia [8]. Experiments in animal models have related the loss of natural teeth to decline in learning and memory functions $[13,14]$. In addition, Onozuka et al. (1999) [15] reported a significant relationship between the decline of chewing function, the degeneration of neurons in the hippocampus, and impairment of spatial memory in elderly mice. This neuronal degeneration in the hippocampus has been identified as a possible cause for the age-related cognitive impairment [16].

Clinical studies have found that the number of teeth present, the median occlusal contact area, and the average value of maximum bite force are lower for elderly with cognitive impairment compared to elderly with normal cognitive function 
$[17,18]$. Studies also indicated an association between a reduced number of teeth and increased risk of developing dementia [19-21] and AD [10, 18]. These data suggest that maintaining dental health may be a non-pharmacological approach to prevent senile dementia and other disorders involving cognitive impairment, such as $\mathrm{AD}[16]$.

Chewing can be objectively assessed by several methods, such as bite force, mandibular movement pattern, and chewing tests. Masticatory performance (MP) is a chewing test that evaluates the extent of grinding of a natural or silicon test food after a standardized number of chewing cycles, either by visual inspection or sieve fractionation [22]. No published studies have evaluated objective measures of chewing in patients with dementia. Reports have estimated that patients with impaired cognitive function also have impaired chewing due to having fewer teeth compared to control groups $[17,18]$, but how cognitive impairment and chewing are related remains unclear.

Considering that there is no cure or prevention for $\mathrm{AD}$ [23], it is important to identify possible risk factors, especially if they are modifiable. Thus, this study aimed to objectively describe masticatory performance in elderly with $\mathrm{AD}$, compared to a cognitively healthy control group.

\section{Material and methods}

\section{Subjects}

This cross-sectional study included two groups of elderly: AD ( $n=16,8$ men and 8 women, $76.7 \pm 6.3$ years) and control ( $n=16,8$ men and 8 women, $75.23 \pm 4.4$ years). All volunteers were elderly, partially or totally edentulous, removable prostheses users and did not display parafunctional habits. Subjects were excluded if they had severe periodontal diseases, physical or cognitive limitations other than $\mathrm{AD}$, or temporomandibular disorders, verified by the Research Diagnose Criteria for Temporomandibular Disorders (RDC/TMD) [24]. An a priori power analysis indicated that 12 subjects per group would yield a power $\beta=0.8$ and $\alpha=0.05$ with a difference of 2 points between groups on MP values. To compensate for possible attrition, 16 individuals were selected for each group.

AD participants were selected from the Program of Cognitive and Functional Kinesiotherapy in Elderly with Alzheimer's Disease (PRO-CDA) at Paulista State University "Julio de Mesquita Filho" (Rio Claro, São Paulo, Brazil). Subjects were also recruited from the Brazilian Alzheimer's Association (ABRAZ, Piracicaba, São Paulo, Brazil). To be included in the $\mathrm{AD}$ group, subjects had to be diagnosed in the mild stage of $\mathrm{AD}$ by a neurologist using the International Classification of Diseases (ICD-10), Diagnostic and Statistical Manual of Mental Disorders (DSM-IV), Mini-Mental State Examination (MMSE), and Clinical Dementia Rating scale (CDR). Individuals were required to have a CDR score of 1.0 or less, indicating that any cognitive impairment present did not exceed the level of mild dementia [25].

The control group was composed of elders seeking prosthetic treatment at Piracicaba Dental School, University of Campinas. Control subjects did not have AD, any other dementia, major depression, or any other mood disorder. Cognitive function of controls was evaluated via MMSE prior to selection. Each group was composed of 11 totally edentulous and five partially edentulous subjects. Figure 1 depicts a flowchart of the patient selection process.

The research protocol was approved by the Ethics Committee of Piracicaba Dental School (\#43/2013), University of Campinas. Patients and their families or legal representatives provided signed written informed consent.

After selection, clinical examinations were performed to objectively assess oral health of all subjects. The clinical examination was performed using a probe, mouth mirror, and flashlight to evaluate each subject's teeth, removable prostheses, and presence of oral pathologies, such as ulcerations and prosthetic stomatitis.

Occlusal supports were recorded by using to the Eichner Index [26]. According to this index, each posterior contact area, including both the premolar and molar regions, are counted as one zone, summing a total of four supporting zones. The molar and premolar contacts of the residual teeth define the classification as follows: (Eichner A): occlusal contacts in all four posterior support zones, (Eichner B): occlusal contacts in three to one zone(s) of contact or in the anterior region only, and (Eichner $C)$ : no occlusal contact at all. Kennedy Classification was also recorded for the partially edentulous subjects [27]. Sociodemographic data relating to educational level and monthly family income were also collected during anamneses.

All study participants received a general dental treatment addressing their individual needs, including periodontal and dental care for their remaining teeth. To standardize prosthetic and oral status, all volunteers received new maxillary and mandibular dentures, which were prepared with acrylic resin using conventional techniques [27]. One experienced dental technician performed all prosthetic procedures. Occlusal denture support was established through the first molars, and a bilateral balanced occlusal scheme was used. Cobalt-chromium alloy (Dentorium; Labordental) was used to process removable partial denture frameworks. The design consisted of a major bar, rests, and clasp retainers adjusted based on each subject's support tissues and remaining teeth. After subjects reported no more chewing complaints, a 2-month period was allowed for complete adaptation to new prosthetics before masticatory performance was evaluated.

\section{Masticatory performance}

Optocal artificial food test based on Optosil polydimethylsiloxane putty (Optosil Comfort, Heraeus Kulzer) was used to 


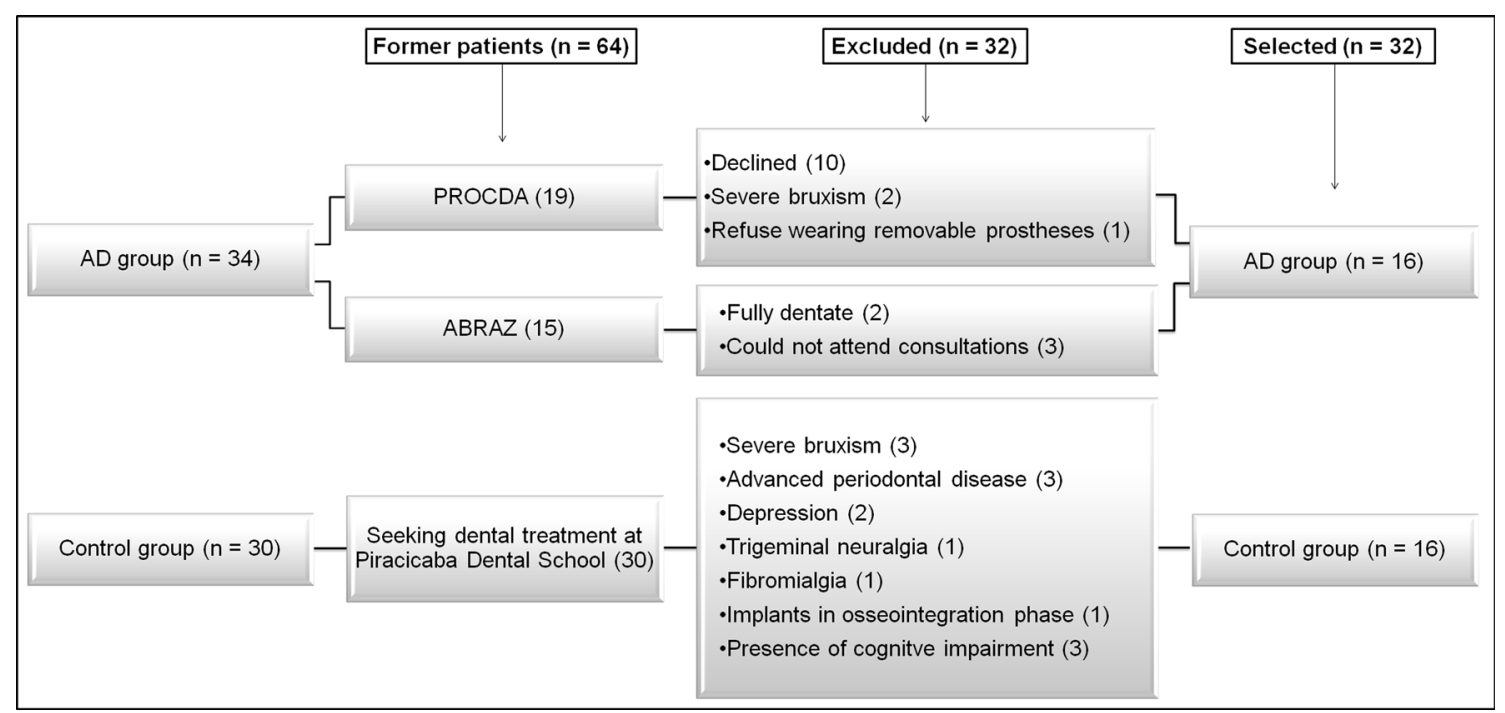

Fig. 1 Flowchart of patient recruitment

prepare cubes measuring $5.6 \mathrm{~mm}$ on each edge [28, 29]. Portions of 17 cubes (approximately $3.7 \mathrm{~g}$ ) were offered to subjects, who were instructed to chew them in a habitual manner. After 40 chewing strokes [28], counted by the examiner, particles were spit onto a paper filter in a glass container, and the subjects rinsed with $200 \mathrm{~mL}$ of water to completely cleanse the oral cavity. The comminuted particles were recovered and dried at room temperature for 1 week, then sieved in a sieving machine (Bertel Indústria Metalúrgica) through a stack of up to 10 sieves for 20 min using gradually decreasing mesh from 5.6 to $0.5 \mathrm{~mm}$. The particles retained on each sieve and in the bottom pans were weighed on a $0.001 \mathrm{~g}$ analytical balance (Mark, BEL Engineering). Masticatory performance was determined according to the median particle size $\left(X_{50}\right)$ calculated using the Rosin-Rammler cumulative function $[28,29]$. High values of $X_{50}$ indicate greater difficulty in grinding the test food material, indicating impaired chewing function.

Instructions were provided to all patients prior to starting the test, and reinforcing instructions were given throughout the test to AD subjects.

\section{MMSE}

Cognitive functioning was assessed using the MMSE [30], which was administered directly to each patient by a trained examiner during the initial research consultation. Although the MMSE had been previously administered by a neurologist for the purpose of diagnosing $\mathrm{AD}$, it was administered a second time by the researcher to correlate values with mastication. This instrument consists of questions grouped into seven categories, designed to evaluate specific cognitive abilities: time orientation (5 points), spatial orientation (5 points), word record ( 3 points), attention and calculation ( 5 points), recall of three words (3 points), language ( 8 points), and visual constructive capacity (1 point). MMSE scores range from a minimum of 0 to a maximum of 30 points with lower scores indicating greater cognitive impairment. In Brazil, the MMSE was translated by Bertolucci et al. (1994) [31], who demonstrated that a cut-off point of 23/24 shows excellent sensitivity and specificity for diagnosis of dementia. Volunteers in the control group with MMSE scores below the cut-off point were excluded from this study and referred to a neurologist for evaluation.

\section{Statistical analyses}

Data were normally distributed according to the Shapiro-Wilk test. Thus, a $t$ test was performed to compare $\mathrm{AD}$ and control groups. A Pearson correlation was performed to assess the correlation between masticatory performance and cognitive status. Power analyses were conducted for all statistical tests. Analyses were performed using SAS version 9.3 (SAS Institute, Inc., Cary, NC) with a significance level of 0.05 .

\section{Results}

No group differences were found in educational level (AD, $4.31 \pm 5.41$ years; control, $4.05 \pm 3.20$ years), monthly family income (AD, $2.41 \pm 2.27$; control, $2.69 \pm 2.66$ ), number of remaining teeth $(\mathrm{AD}, 4.31 \pm 6.82$; control, $3.94 \pm 6.54)$. The number of subjects with total and partial dentures in each group was balanced, so no differences were detected. Subject's dental status was presented according to their removable prostheses type, Kennedy Classification and Eichner Index (Table 1). 
Table 1 Number of subjects according to their removable prostheses type, Kennedy Classification and Eichner Index in $\mathrm{AD}$ and control groups

\begin{tabular}{lcc}
\hline & Groups & \\
\cline { 2 - 3 } & AD & Control \\
\hline Totally edentulous & & 10 \\
Using upper and lower CD & 10 & 1 \\
Using upper CD and lower implant supported overdenture & 1 & \\
Partially edentulous & & 2 \\
Using upper CD and lower RPD & 2 & 3 \\
Using upper and lower RPD & 2 & 0 \\
Using upper implant supported overdenture and lower RPD & 1 & 1 \\
Kennedy classification & & 1 \\
Mandibular class I and edentulous maxilla & 1 & 1 \\
Mandibular class II and edentulous maxilla & 2 & 1 \\
Mandibular and maxillary class I & 1 & 1 \\
Mandibular and maxillary class II & 1 & 14 \\
Mandibular and maxillary class III & 0 & 2 \\
Eichner Index & & \\
A & 1 & \\
B & 1 & \\
C & 0 & 15 \\
\hline
\end{tabular}

$C D$ complete denture; $R P D$ removable partial denture
Compared to controls, AD subjects had decreased MMSE values and masticatory performance, expressed as $X_{50}$ values, as shown in Table 2. There was a moderate negative correlation between masticatory performance and cognitive parameters (Pearson's $r=-0.69, P<0.001$; power $\beta=0.83$ ). Thus, greater MMSE scores were associated with lower $X_{50}$ values, indicating greater chewing deficits.

\section{Discussion}

This study was the first to investigate the association between masticatory function and cognitive status of mild AD patients compared to controls. A moderate correlation was discovered, indicating that both cognitive and masticatory function decline in mild $\mathrm{AD}$.

Elderly subjects in the present study had sociodemographic characteristics comparable to previous reports [32, 33],

Table 2 Mean values (standard deviation) for masticatory performance $\left(X_{50}\right)$ and MMSE for AD and control subjects

\begin{tabular}{|c|c|c|c|c|}
\hline & \multicolumn{2}{|l|}{ Groups } & \multirow[b]{2}{*}{$P$ value } & \multirow[b]{2}{*}{ Power $\beta$} \\
\hline & $\mathrm{AD}$ & Control & & \\
\hline$X_{50}$ & $6.36 \pm 1.01$ & $4.34 \pm 1.16$ & $<0.001$ & $>0.999$ \\
\hline MMSE & $17.92 \pm 7.51$ & $26.85 \pm 1.68$ & 0.001 & $>0.999$ \\
\hline
\end{tabular}

Data were analyzed using $t$ tests including age, years of education, monthly income, MMSE scores, percentage of edentulism, and number of remaining teeth. The control group showed higher MMSE values, which is expected in a cognitively preserved population [34]. Studies comparing $\mathrm{AD}$ patients to healthy control groups have found better oral health in $\mathrm{AD}[32,33,35]$. However, different from previous studies, we objectively assessed masticatory performance. Thus, care was taken to select $\mathrm{AD}$ and control subjects with the same types of prostheses and numbers of remaining teeth, since those aspects could bias the study by influencing chewing function [36].

Previous studies $[8,37,38]$ found impaired chewing function in $\mathrm{AD}$ patients, but attributed this to their dental condition and decreased number of remaining teeth $[37,38]$ and used subjective measurements [8]. Although dental conditions strongly influence masticatory performance [36], our data show that chewing in mild $\mathrm{AD}$ is impaired even compared to elderly with similar age and oral characteristics, demonstrating a deleterious effect of the disease on mastication. Data on masticatory performance obtained from our control group support previous studies evaluating totally or partially edentulous patients in the same age range [39, 40].

The association between tooth loss, reduction in masticatory function, and cognitive decline in patients with $\mathrm{AD}$ has been previously demonstrated $[9,10,21]$. Chewing may stimulate brain areas related to memory, especially the hippocampus, which could explain how efficient chewing prevents cognitive impairment [13-16]. On the other hand, AD can impair masticatory function by reducing muscle function and appetite [41]. 
Thus, the disease can worsen masticatory function, while the reduction in chewing function due to tooth loss could lead to cognitive decline.

Mastication is a complex process controlled by the central nervous system and that can be influenced by several subject factors, such as age, dental status, and gender, and is dependent in large part on sensory feedback [42]. Smell, taste, texture, and hardness of the food also influence the masticatory cycle [42]. The neuronal damage and brain atrophy present in Alzheimer's disease also affect the area corresponding to the chewing, the cortico-bulbar tract, which can bring impacts on masticatory function [2]. Furthermore, the senses of smell and taste are known to be adversely affected by the disease, which possibly impairs sensory feedback necessary for mastication [2]. In addition, bite force also shows a positive relationship with masticatory performance and tends to reduce with aging due to the atrophy of the jaw-closing muscles, even more evident in edentulous than in dentate subjects [43]. Since skeletal muscle atrophy, declining strength, and physical frailty can be present in $\mathrm{AD}$, it is possible that elderly with $\mathrm{AD}$ presents an even more impaired bite force and that should be a possible cause for their decreased chewing function.

In spite of our relatively small number of participants, the power of all statistical tests was greater than 0.8 . Moreover, high levels of statistical significance were found, showing reliability of results. Thus, our data show that cognitive impairment is associated with decreased masticatory function. The presence of chewing impairment in AD patients should encourage dentists to pay special attention to this group and to improve their chewing function by providing well fit prostheses with appropriate occlusal contacts.

\section{Conclusion and clinical relevance}

The role of the clinicians in the $\mathrm{AD}$ patient's treatment is similar to that applied to elders without the disease, such as to maintain a healthy oral function and minimize the progression of oral diseases such as dental caries and periodontal diseases. However, it is important to emphasize that efforts should be made to maintain natural teeth in the mouth whenever possible, since natural teeth provide a better masticatory function than removable dentures, and also due to the possible cognitive impairment associated with tooth loss. With regard to oral rehabilitation, most of elders still need removable prostheses [44]. Thus, partial removable prosthesis should be planned to provide an easy axis to insert and remove, but stable. The denture's stability can be obtained with functional molding procedures and additionally providing a balanced occlusion [45]. The prostheses adjustment should be done regularly, regardless of patients complaints or not, since one may not be able to realize when prosthesis is hurting. The frequency of monitoring consultation should be greater than that of an elderly without cognitive deficit.
Another aspect to note is regarding the hygiene procedures of the prosthesis and remaining teeth, which often need to be performed or supervised by a caregiver. The caregiver should be instructed by the dentist on how to do it. Family members should be informed about reduction in chewing capacity to make adjustments in diet to allow the elderly to eat comfortably without nutritional losses.

Acknowledgments This study was supported by the National Council of Technological and Scientific Development (CNPq, grant number 48.090.3/2013-1) and by São Paulo Research Foundation (FAPESP, grant number 2013/10200-7), Brazil.

Compliance with ethical standards The research protocol was approved by the Ethics Committee of Piracicaba Dental School (\#43/ 2013), University of Campinas. Patients and their families or legal representatives provided signed written informed consent.

Conflict of interest The authors declare that they have no conflicts of interest.

Ethical approval All procedures were in accordance with the ethical standards of the institutional and/or national research committee and with the 1964 Helsinki declaration and its later amendments or comparable ethical standards.

Informed consent Informed consent was obtained from all individual participants included in the study.

\section{References}

1. Ewbank DC (1999) Deaths attributable to Alzheimer's disease in the United States. Am J Public Health 89(1):90-92

2. Friedlander A, Norman D (2006) Alzheimer's disease psychopathology, medical management and dental implications. Am Dent J 137(9):1240-1251

3. Reiman EM, Caselli RJ (1999) Alzheimer's disease. Maturitas 31(3): 185-200

4. Braak E, Griffing K, Arai K, Bohl J, Bratzke H, Braak H (1999) Neuropathology of Alzheimer's disease: what is new since A. Alzheimer? Eur Arch Psychiatry Clin Neurosci 249(Suppl):14-22

5. Thal DR, Griffin WST, Braak H (2008) Parenchymal and vascular A beta-deposition and its effects on the degeneration of neurons and cognition in Alzheimer's disease. J Cell Mol Med 12(5B):18481862

6. Cummings JL, New T, Journal E, Jul B (2010) Document ViewProQuest Alzheimer 's disease document view_ProQuest 351(1): $1-11$

7. Mattson MP (2004) Pathways towards and away from Alzheimer's disease. Nature 430(7000):631-639

8. Lexomboon D, Trulsson M, Wårdh I, Parker MG (2012) Chewing ability and tooth loss: association with cognitive impairment in an elderly population study. J Am Geriatr Soc 60(10):1951-1956

9. Okamoto N, Morikawa M, Okamoto K, Habu N, Hazaki K, Harano A et al (2010) Tooth loss is associated with mild memory impairment in the elderly: the Fujiwara-kyo study. Brain Res 19; 1349:68 75

10. Gatz M, Mortimer JA, Fratiglioni L, Johansson B, Berg S, Reynolds CA, et al. (2006) Potentially modifiable risk factors for dementia in identical twins. Alzheimers Dement 2(2):110-117 
11. Bettens K, Sleegers K, Van Broeckhoven C (2010) Current status on Alzheimer disease molecular genetics: from past, to present, to future. Hum Mol Genet 19(R1):R4-11

12. Müller-Spahn F, Hock C (1999) Risk factors and differential diagnosis of Alzheimer's disease. Eur Arch Psychiatry Clin Neurosci 249(S3):S37-S42

13. Oue H, Miyamoto Y, Okada S, Koretake K, Jung C-G, Michikawa $\mathrm{M}$ et al (2013) Tooth loss induces memory impairment and neuronal cell loss in APP transgenic mice. Behav Brain Res 1; 252:318-325

14. Oue H, Miyamoto Y, Koretake K, Okada S, Doi K, Jung CG, et al. (2014) Tooth loss might not alter molecular pathogenesis in an aged transgenic Alzheimer's disease model mouse. Gerodontology. doi:10.1111/ger.12153

15. Onozuka M, Watanabe K, Mirbod SM, Ozono S, Nishiyama K, Karasawa N, et al. (1999) Reduced mastication stimulates impairment of spatial memory and degeneration of hippocampal neurons in aged SAMP8 mice. Brain Res 826(1):148-153

16. Ono Y, Yamamoto T, Kubo K, Onozuka M (2010) Occlusion and brain function: mastication as a prevention of cognitive dysfunction. J Oral Rehabil 37(8):624-640

17. Miura H, Kariyasu M, Yamasaki K, Arai Y, Sumi Y (2005) Relationship between general health status and the change in chewing ability: a longitudinal study of the frail elderly in Japan over a 3-year period. Gerodontology 22(4):200-205

18. Kondo K, Niino M, Shido K (1994) A case-control study of Alzheimer's disease in Japan-significance of life-styles. Dementia 5:314-326

19. Stein P, Desrosiers M (2007) Tooth loss, dementia and neuropathology in the Nun study. J Am Dent Assoc 138:1314-1322

20. Kim J, Stewart R, Prince M, Kim S, Yang S, Shin I, et al. (2007) Dental health, nutritional status and recent-onset dementia in a Korean community population. Int J Geriatr Psychiatry 22:850-855

21. Stein PS, Kryscio RJ, Desrosiers M, Donegan SJ, Gibbs MB (2010) Tooth loss, apolipoprotein E, and decline in delayed word recall. J Dent Res 89(5):473-477

22. Boretti G, Bickel M, Geering AH (1995) A review of masticatory ability and efficiency. J Prosthet Dent 74(4):400-403

23. Rolim TDS, Fabri GMC, Nitrini R, Anghinah R, Teixeira MJ, de Siqueira JTT, et al. (2014) Evaluation of patients with Alzheimer's disease before and after dental treatment. Arq Neuropsiquiatr 72(12):919-924

24. Dworkin SF, LeResche L (1992) Research diagnostic criteria for temporomandibular disorders: review, criteria, examinations and specifications, critique. J Craniomandib Disord 6:301-355

25. Clark CM, Ewbank DC (1996) Performance of the dementia severity rating scale: a caregiver questionnaire for rating severity in Alzheimer disease. Alzheimer Dis Assoc Disord 10(1):31-39

26. Ikebe K, Maeda Y, Index E (2010) Validation of the Eichner index in relation to occlusal force and masticatory performance. Int $\mathrm{J}$ Prosthodont 23(6):521-524

27. Carr AB, Brown DT, McCracken WL. McCracken's removable partial prosthodontics, Twelfth Edition. Twelfth. St. Louis, Mo.: Elsevier Mosby; 2011. 400 p.

28. Slagter AP, Bosman F, Van der Bilt A (1993) Comminution of two artificial test foods by dentate and edentulous subjects. J Oral Rehabil 20(2):159-176
29. Pocztaruk RDL, Frasca LCDF, Rivaldo EG, Fernandes EDL, Gavião MBD (2008) Protocol for production of a chewable material for masticatory function tests (Optocal-Brazilian version). Braz Oral Res 22(4):305-310

30. Folstein MF, Folstein SE, McHugh PR (1975) "Mini-mental state". A practical method for grading the cognitive state of patients for the clinician. J Psychiatr Res 12(3):189-198

31. Bertolucci PHF, Brucki SMD, Campacci SR, Juliano Y (1994) O Mini-Exame do Estado Mental em uma população geral: impacto da escolaridade. Arq Neuropsiquiatr 52(1):01-07

32. Ribeiro GR, Costa JLR, Ambrosano GMB, Garcia RCMR (2012) Oral health of the elderly with Alzheimer's disease. Oral Surg Oral Med Oral Pathol Oral Radiol 114(3):338-343

33. Cicciù $M$, Matacena $G$, Signorino $F$, Brugaletta $A$, Cicciù A, Bramanti E (2013) Relationship between oral health and its impact on the quality life of Alzheimer's disease patients: a supportive care trial. Int J Clin Exp Med 6(9):766-772

34. Uhlmann RF, Larson EB (1991) Effect of education on the minimental state examination as a screening test for dementia. J Am Geriatr Soc 39(9):876-880

35. Black BS, Johnston D, Morrison A, Rabins PV, Lyketsos CG, Samus QM (2012) Quality of life of community-residing persons with dementia based on self-rated and caregiver-rated measures. Qual Life Res 21(8):1379-1389

36. Hatch JP, Shinkai RS, Sakai S, Rugh JD, Paunovich ED (2001) Determinants of masticatory performance in dentate adults. Arch Oral Biol 46(7):641-648

37. Miura H, Yamasaki K, Kariyasu M, Miura K, Sumi Y (2003) Relationship between cognitive function and mastication in elderly females. J Oral Rehabil 30(8):808-811

38. Mummolo S, Ortu E, Necozione S, Marzo G (2014) Relationship between mastication and cognitive function in elderly in L'Aquila. Int J Clin Exp Med 7(4):1040-1046

39. Fontijn-Tekamp FA, Slagter AP, Van Der Bilt A, Van 'T Hof MA, Witter DJ, Kalk W, et al. (2000) Biting and chewing in overdentures, full dentures, and natural dentitions. J Dent Res 79(7):1519-1524

40. Slagter AP, Olthoff LW, Steen WH, Bosman F (1992) Comminution of food by complete-denture wearers. J Dent Res 71(2):380-386

41. Kocaelli H, Yaltirik M, Yargic LI, Özbas H (2002) Alzheimer's disease and dental management. Oral Surg Oral Med Oral Pathol Oral Radiol Endod 93(5):521-524

42. Lund JP (1991) Mastication and its control by the brain stem. Crit Rev Oral Biol Med 2(1):33-64

43. Ikebe K, Nokubi T, Morii K, Kashiwagi J, Furuya M (2005) Association of bite force with ageing and occlusal support in older adults. J Dent 33(2):131-137

44. Douglass CW, Shih A, Ostry L (2002) Will there be a need for complete dentures in the United States in 2020? J Prosthet Dent 87(1):5-8

45. Shirani M, Mosharraf R, Shirany M (2014) Comparisons of patient satisfaction levels with complete dentures of different occlusions: a randomized clinical trial. J Prosthodont 23(4):259-266 